\section{A experiência da perimenopausa e pós-menopausa com mulheres que fazem uso ou não da terapia de reposição hormonal}

\author{
Peri-menopausal and post-menopausal \\ experience among women with and without \\ hormone replacement therapy
}

Sônia Maria Garcia Vigeta 1 Ana Cristina Passarella Brêtas 1

\title{
Introdução
}

1 Departamento de Enfermagem, Universidade Federal de São Paulo, São Paulo, Brasil.

Correspondência S. M. G. Vigeta Departamento de Enfermagem, Universidade Federal de São Paulo. Rua Napoleão de Barros 754, São Paulo, SP 04024-002, Brasil. vigeta@uol.com.br

\begin{abstract}
This qualitative study focuses on peri-menopausal and post-menopausal experience and its signs and symptoms for women with and without use of hormone replacement therapy (HRT). Empirical data were obtained through interviews with 11 women (range: 45 to 59 years) with or without HRT at different health clinics in the Municipality of São Paulo, Brazil. The analysis used the hermeneutic method. Most significantly, women who are not on HRT present peri-menopausal symptoms, but they "live with" such symptoms; meanwhile, women in use of isoflavone rely on alternative therapies for the bothersome symptoms; women using HRT report a feeling of wellbeing. The article suggests the importance of training health professionals to understand the peri-menopausal and post-menopausal periods as part of the aging process, and thus as a vital rather than pathological process.
\end{abstract}

Nursing; Aging; Menopause; Hormonal Reposition Therapy; Women's Health
O conhecimento sobre o envelhecimento da população humana constitui um fenômeno relativamente recente na história da humanidade. A média de sobrevida das mulheres na Idade Média era de 25 anos, só no início século XX com os avanços científicos é que a expectativa de vida pôde chegar aos 50 anos. No Brasil, a esperança de vida média ao nascer da mulher brasileira no ano 2001 foi de 68,82 anos 1,2,3.

Com o aumento da expectativa de vida, as mulheres passaram a sobreviver um tempo suficiente para poder experimentar mudanças em seus corpos, o que muitas de gerações anteriores não conseguiram vivenciar.

As mulheres que hoje chegam à menopausa fazem parte da geração baby boom, que é caracterizada por pessoas que nasceram após a Segunda Grande Guerra Mundial. Essa geração foi em grande parte a responsável por mudanças sócio-culturais importantes, especialmente na década de 60 do século passado e estão promovendo uma redefinição da idade madura 4 .

Estudos em que foi questionado à população em geral sobre o que é climatério ou menopausa, verificou-se que a quantidade e a qualidade das informações são muito baixas 5. Pesquisas comparativas mostram que em algumas sociedades não existe um termo, empírico ou científico, equivalente para a menopausa. Os sintomas que são identificados no ocidente po- 
dem estar ausentes em outras sociedades, ou, se presentes, tidos como insignificantes. Podemos, portanto, depreender que a consciência e a percepção social da imagem do corpo não são universais $6,7,8$.

O relatório do grupo técnico de pesquisa sobre Menopausia en los Años Noventa 9 , publicado pela Organização Mundial da Saúde (OMS) em 1996, menciona que já em 1980, este órgão internacional recomendava que fossem investigadas as características e os efeitos da menopausa nas mulheres dos países em desenvolvimento; entretanto, pouco foi feito. Solicitava ainda a utilização de termos definidos para a fase da menopausa a fim de que fosse possível comparar resultados de trabalhos publicados em diferentes lugares do mundo, visando formar um consenso técnico. Sugeria também o abandono do termo climatério que, embora consagrado pelo uso, tem gerado ambigüidades no meio científico.

A OMS 9 recomenda a utilização dos termos: "menopausa natural" para o evento da parada permanente da menstruação, que é resultante da perda da atividade folicular dos ovários e só é reconhecido retrospectivamente após um ano de amenorréia, sem outra causa patológica ou psicológica; "perimenopausa" ou "climatério" para o período em que surgem as irregularidades menstruais e queixas vasomotoras, que antecedem a menopausa e vão até o primeiro ano seguinte a ela; "transição menopáusica” é o termo relacionado ao que na prática equivale a perimenopausa; "pré-menopausa" é o período total reprodutivo, anterior à menopausa; “pósmenopausa" corresponde ao período após o evento da menopausa, independente de a menopausa ter sido natural ou induzida e se prolonga até uma idade avançada. Compreende-se que esse limite se dê por meio da homeostase hormonal que ocorre na velhice, quando a carência estrogênica fica compensada pela perda progressiva dos receptores estrogênicos 9,10.

O primeiro estudo brasileiro sobre menopausa foi realizado no Município de Campinas, Estado de São Paulo, que caracterizou as usuárias de terapia de reposição hormonal (TRH), tratou-se de um estudo descritivo de corte transversal, tipo inquérito populacional domiciliar, que veio mostrar que entre as principais características das mulheres que se submetem a esta terapia é estar na perimenopausa e apresentar sintomas climatéricos, especificamente as ondas de calor. Constatou-se que apresentavam um estilo de vida mais saudável, melhor classe social, melhor nível educacional e utilizavam os serviços médicos preventivos 11 .

A segurança da TRH para as mulheres ficou abalada após a publicação parcial e interrupção do estudo americano multicêntrico, duplocego, controlado com placebo e randomizado, realizado por pesquisadores do Women's Health Initiative (WHI). Os riscos globais excediam os benefícios, houve um aumento significativo do risco de doença arterial coronariana, de acidente vascular cerebral, de tromboembolismo venoso; um decréscimo significativo do risco de câncer de cólon e do risco total de fraturas e, quanto ao câncer de mama, apresentou uma relação de risco em razão do tempo de duração da terapia 12,13 .

Nas últimas duas décadas, as pesquisas têm privilegiado a busca de terapias capazes de aliviar os sintomas indesejáveis associados às mudanças hormonais, sobretudo o desenvolvimento de compostos farmacêuticos de hormônios sintéticos. Porém, pouco ou quase nunca as mulheres são ouvidas pelos pesquisadores e/ou profissionais da área da saúde sobre como se sentem nesta fase e como gostariam de ser cuidadas.

Assim, este trabalho teve por objetivo conhecer o significado da perimenopausa e o vivenciar de seus sinais e sintomas para mulheres que usam ou não a TRH.

\section{Metodologia}

Este estudo foi construído na abordagem qualitativa, baseando-se na premissa de que existe uma relação dinâmica entre o mundo real e o sujeito, entre o sujeito e o objeto, entre o mundo objetivo e a subjetividade do sujeito. Segundo Minayo 14, ao contemplarmos o aspecto qualitativo do objeto estamos considerando como sujeito de estudo: pessoas, com características próprias, pertencentes a determinado grupo/ classe social com suas crenças, valores e significados. Está ancorado teoricamente no paradigma da pós-modernidade que privilegia a não-dicotomia entre as ciências, voltando a reflexão epistemológica para a análise dos modelos relacionais da investigação científica 15 .

\section{Procedimentos metodológicos}

Vale lembrar que os procedimentos éticos inerentes a pesquisas desta natureza, bem como a vigilância rigorosa das condições de utilização 
das técnicas e a sua adequação ao problema posto, estiveram presentes em todas as etapas deste estudo. O projeto de pesquisa foi aprovado pelo Comitê de Ética em Pesquisa da Universidade Federal de São Paulo/Hospital São Paulo, sob o número 1.219/02.

(a) Local do estudo: inicialmente as entrevistas foram realizadas em dois serviços de saúde pública destinados à assistência de mulheres na perimenopausa e pós-menopausa, com a organização de trabalho centrada no profissional médico. A maioria das pesquisadas nestes locais eram usuárias da TRH. Levando-se em conta que para desnudar o objeto do estudo precisaríamos ouvir também mulheres que não utilizavam a TRH, optamos por buscá-las em órgãos vinculados ao movimento feminista. Tal escolha se pautou na crença de que mulheres freqüentadoras e/ou militantes deste movimento teriam maior conhecimento e controle sobre os seus próprios corpos, não deixando livremente a escolha da terapêutica à mercê de decisão médica. Por fim, preferimos trabalhar com a técnica da "bola de neve", em que cada depoente indicava outra.

(b) População do estudo: foi composta por 11 mulheres na faixa etária de 45 a 59 anos de idade, sendo quatro usuárias da TRH clássica e sete não usuárias, e três destas eram usuárias de isoflavona. Concordaram em participar da pesquisa após terem sido informadas sobre todas as etapas, assim como sobre os objetivos, finalidades e formas de divulgação dos resultados. Feito isso, assinaram o Termo de Consentimento Livre e Esclarecido.

O número de sujeitos, em virtude da natureza qualitativa do estudo, foi definido durante o processo de coleta de dados. Esta escolha se sustentou em Thiollent 16, quando menciona que a utilização de técnica de entrevista em uma pesquisa qualitativa não requer determinação amostral para a definição dos sujeitos que serão entrevistados. A seleção dos narradores se baseia na disponibilidade do entrevistado, a qual não é previsível antes de um primeiro contato. A seleção resulta de uma avaliação da relevância ou da representatividade social (não estatística) das pessoas. Tal avaliação fica por conta da sensibilidade e conhecimento técnico-científico do pesquisador.

Vale destacar que a finalidade de entrevistar estes sujeitos foi a de consubstanciar o objeto do estudo, uma vez que estas mulheres já vivenciavam a perimenopausa ou a pós-menopausa; logo, possuíam subsídios empíricos suficientes para discorrer sobre esta fase, relatando seus sentimentos, atitudes, valores, contribuindo para a construção desta pesquisa.
Este distanciamento do objeto com a introdução de olhares de outros sujeitos Bourdieu 17 denomina de objetivação participante, e reforça que essa abordagem faz com que o pesquisador passe a ter uma visão global do estudo apreendido, fundamentalmente porque saiu dele.

(c) Coleta de dados: foi obtida por meio da técnica da entrevista realizada com roteiro semiestruturado. Foram gravadas individualmente por uma das pesquisadoras, cabendo a ela a transcrição textual, como forma de assegurar o sigilo acordado com as entrevistadas, mantendo a construção ética do trabalho.

Às depoentes foi conferida a posição de narradoras, pois o que nos interessava era que deixassem fluir o pensamento e, com isso, contassem suas experiências, auxiliando na construção do imaginário coletivo referente à vivência da perimenopausa em situação de uso ou não da TRH. Foram tratadas como informantes do objeto em estudo, e não como objetos de análise intensiva.

Para efeito de análise mantivemos a distinção entre as entrevistadas, colocando após a citação das narrativas um número correspondente a cada uma delas, seguido pela sua idade e pela identificação do tipo de terapia que utiliza: S/H (sem TRH), S/H/I (sem TRH com isoflavona), $\mathrm{C} / \mathrm{H}$ (com $\mathrm{TRH})$.

(d) Análise dos dados: foi realizada à luz da hermenêutica. Optamos por sua utilização porque esse "caminho de pensamento", como afirma Minayo 14 (p. 227), faz com que o pesquisador "busque entender o texto, a fala, o depoimento como resultado de um processo social (trabalho e dominação) e o processo de conhecimento (expresso em linguagem) ambos frutos de múltiplas determinações mas com significado específico".

Para viabilizar a utilização da hermenêutica no processo de análise dos dados obtidos didaticamente, percorremos os seguintes passos: (1) ordenação dos dados obtidos por meio das entrevistas, da observação de campo e dos referenciais bibliográficos;

(2) classificação dos dados obtidos nos textos (empíricos e teóricos) pela leitura rigorosa e crítica, buscando relacionar as categorias analíticas (teóricas) e as empíricas;

(3) reordenação das entrevistas, adotando o recurso proposto por Bourdieu 17 para análise relacional, em que se inscreve cada uma das entrevistas em uma linha e abre-se uma coluna sempre que se descobre uma propriedade necessária para caracterizar uma delas, o que nos obriga a interrogar sobre a presença ou ausência dessa propriedade em todas as outras (Fase indutiva); (4) reclassificação do material obtido na fase indutiva, unindo as colunas que registraram ca- 
tegorias equivalentes, de maneira a reter todas as características presentes nos textos, relevantes para apreensão do objeto em estudo. Para reclassificar este material objetivando a sua análise, foram criadas duas unidades temáticas: (1ㅇ) o conhecimento e percepção dos sinais e sintomas da perimenopausa no corpo em processo de envelhecimento e (2ㅇ) o conhecimento sobre propostas terapêuticas;

(5) realização de análise crítica, relacional, entre os dados empíricos obtidos nas entrevistas e categorias analíticas selecionadas.

\section{Resultados e discussão}

A discussão dos dados será apresentada por intermédio das unidades temáticas, que foram construídas valendo-se do conteúdo obtido nas narrativas.

\section{Unidade temática I: o conhecimento e percepção dos sinais e sintomas da perimenopausa no corpo em processo de envelhecimento}

Das mulheres entrevistadas, encontramos em suas narrativas uma percepção maior sobre o processo do envelhecimento na época da menopausa. Colocaram que o envelhecer acontece de forma individual para cada mulher, dependendo do seu autocuidado, de uma auto-imagem positiva, podendo continuar a ser atraente durante toda a vida.

Há um reconhecimento maior das mulheres sem TRH e das sob o uso da isoflavona, do que seja a perimenopausa. Mostram ter conhecimento de que é um período em que a mulher apresenta alguns meses com irregularidade menstrual ou pára a menstruação definitivamente, e que ocorre aproximadamente aos cinqüenta anos de idade. Não houve relato do conhecimento da necessidade de esperar o tempo de 12 meses para se certificarem de que estejam na menopausa; entendem o período como uma fase em que há diminuição ou total falta de hormônios, que conseqüentemente as impede de reproduzir na espécie humana e produz em algumas mulheres sintomas típicos.

"Coisa de oito anos, por aí mais ou menos. (...) É pelo calor (...) a minha menstruação ia e voltava, ia e voltava (...). Tinha uma época que eu fiquei nove meses sem vir; (...). Não, a primeira vez acho que faltou uns quatro ou cinco meses. Aí eu fui ao médico que falou que era assim mesmo. Depois eu já esqueci, eu nem ligava mais que faltava eu nem ligava mais" (4, $59, \mathrm{~S} / \mathrm{H})$.
Foi a partir de 1920 que o modelo biomédico passou a definir a menopausa como escassez da produção do estrogênio, terminando por constituir-se numa doença de privação hormonal reforçada pelas inúmeras publicações especializadas ou leigas.

"Mas eu acho que uma pessoa, não é porque entrou na menopausa, que ela é uma pessoa doente, não é. Apenas passou uma fase da vida dela..." (10, 59, S/H).

Nas narrativas das mulheres sob a ação da TRH encontramos relatos diversos desde as que não se perceberam na perimenopausa por falta de conhecimento, ou por terem vivenciado uma menopausa induzida cirurgicamente, em que ocorre introdução da terapêutica sem que a mulher perceba os sintomas.

"Bom, eu realmente não li nada. Porque eu achei que eu não estivesse nessa época ainda. Quando eu cheguei aqui é que a médica mandou eu fazer uma série de exames. E eu percebi, ou melhor, ela percebeu que eu precisava fazer uma reposição leve hormonal" (1, 54, C/H).

Verificamos na narrativa da depoente a declaração de que submete seu corpo ao poder médico. Como entender tal dominação? Concordamos com Foucault 18 quando afirma que não há poder, mas sim práticas ou relações de poder disseminadas por toda estrutura social. Atingindo o corpo dos indivíduos, penetrando em suas vidas cotidianas, nos seus gestos, atitudes, comportamentos, hábitos, discursos, criando uma rede de micropoderes, e nada ou ninguém escapa dessa rede; por conseguinte, nada está isento de poder.

Acreditamos que uma maneira de minimizar tal constatação seja trabalhar com educação para a saúde, instrumentalizando as mulheres para compreenderem o funcionamento do próprio corpo, estabelecendo uma relação mais equânime entre o saber e o poder. Nessa dimensão, visualizamos o trabalho dos profissionais de saúde, entre eles o do enfermeiro.

No atendimento à saúde integral da mulher enquanto política de saúde pública, é dada uma maior ênfase à assistência à mulher na sua fase reprodutiva, fértil. E há poucos serviços públicos ou privados de saúde que se preocupem em esclarecer as mulheres sobre a fase da menopausa.

As mulheres que não fazem uso da TRH e vivenciaram pessoas próximas na menopausa sem sintomatologia apresentam um entendimento da menopausa como um processo fisiológico; todavia, aquelas que presenciaram a menopausa de outra pessoa com sintomas, procuram se auto-educar a fim de vivenciá-los de outra maneira que não cause constrangimentos, 
ou melhor, que os outros não percebam que está tendo os sintomas da menopausa.

As mulheres com o uso da TRH revelam a existência de mulheres que apresentam sintomas mas não procuram assistência médica por vários motivos, desde econômicos até educacionais, no entanto, elas relatam que procuram tratamento e que não gostam de comentar com outras mulheres sobre sua passagem pelo evento da menopausa.

A menopausa é ainda considerada um assunto tabu, por ser constrangedor, por ser íntimo e ser relacionado à sexualidade. Essa é uma visão socialmente construída da menopausa, que vem com uma conotação negativa de perdas.

Os sinais e sintomas que se apresentam como consenso da decorrência da diminuição dos níveis hormonais são os sintomas vasomotores (ondas de calor) e os atróficos (principalmente a atrofia vaginal).

“Os calores, essas coisas, que eu saiba, ela não teve [referindo-se à sua mãe], coisas que eu e minha irmã temos (...) porque mesmo no frio, dá calor, então deve ser" (7, 59, S/H/I).

Classicamente as ondas de calor acontecem à noite, conseqüentemente, tendem a afetar a qualidade do sono, promovendo distúrbios psíquicos como fadiga e irritabilidade, além da diminuição do poder de concentração e da memória.

"Teve um negócio no sono com a menopausa. (...). É que você deita e o sono não vem. (...) você está detonada no dia seguinte, é horrível. E é uma falta de sono, que você saca que é falta de hormônio mesmo. Diferente de um sono, que você não dorme por ansiedade, que é uma noite ou outra, era uma constante mesmo" (9, 51, S/H/I).

Fortes \& Hirata 19 acreditam que muitos dos sintomas psíquicos atribuídos à transição menopáusica como a depressão, ansiedade, irritabilidade e modificações da libido, na mulher emocionalmente estável, não sofram modificações. Enquanto outros estudiosos como Baggio 20 admitem que as alterações hormonais e somáticas próprias deste período possam gerar distúrbios emocionais mesmo em mulheres consideradas sadias do ponto de vista emocional.

"Eu sentia (...) mas eu não sei se era da menopausa, depois fiquei sabendo que era do lúpus, muita depressão, tinha muita vontade assim de, ... eu não queria mais viver... porque tentei o suicídio três vezes, (...)" $(5,45, \mathrm{C} / \mathrm{H})$.

É, pois, necessário, na anamnese da mulher na perimenopausa, afastar muitos dos sintomas apresentados que podem ser de outras patologias, por ser comum nessa faixa etária o aumento na incidência de doenças crônicas, como hipertensão, diabetes, hipotireoidismo e outras.

A atrofia vulvovaginal por hipoestrogenismo ocasiona a perda da rugosidade da mucosa vulvovaginal com redução acentuada da lubrificação (secura vaginal) e modificações importantes da flora vaginal nativa, levando conseqüentemente ao aparecimento de prurido vulvar e a dispareunia 21.

"Quer dizer, eu acho que estou ficando mais seca, (...). Mas também não no ponto de doer, (...) vagina diminui(...), é muito estranho, é muito louco isso" (9, 51, S/H/I).

As mulheres entrevistadas sem uso da TRH, com uso da isoflavona ou com uso da TRH são quase unânimes em afirmar que há uma diminuição na freqüência das relações sexuais em relação ao passado, quando eram mais jovens.

No entanto, a diminuição de lubrificante vaginal, que é vista muitas vezes como uma alteração patológica, não se configura, para as mulheres, um fator que as impeçam de ter uma relação sexual satisfatória. Relatam que aumenta o tempo para que se sintam úmidas, mas não apresentam dor no intercurso sexual.

“Não sei, eu acho que sou a mesma coisa, não sou muito chegada a ficar, sabe ..., entre marido e mulher. (...) mais nova sim, mas agora não. Mas é que ele continua o mesmo, mais velho que eu, mas só que eu ..., Dói? Não dói, mas acho que é problema da menopausa (....)" (4, 59, S/H).

Trien 22 relata que as alterações na anatomia e funcionamento sexual são naturais e acontecem com todas as pessoas com o avanço da idade. E que vários pesquisadores em seus estudos, entre eles Masters e Johnson, colaboraram para concluir que: "se você não usa o sexo, você o perde". Apregoam que a atividade sexual regular, pelo menos uma ou duas vezes por semana, mantém a vagina úmida e elástica, mas pode ser substituída por qualquer espécie de estímulo sexual que propicie o aumento do fluxo sangüíneo para a região, seja sob a forma de fantasias, masturbação ou intercurso.

As mulheres que recorrem à isoflavona apresentam os sintomas na menopausa que as incomodam e, como há contra-indicações da TRH clássica, ou não querem assumir, buscam terapias alternativas. Na homeopatia procuram solução para a insônia e depressão que a isoflavona não parece resolver. A atrofia vaginal e secura vaginal são citadas como sintomas que existem mesmo com o uso da isoflavona, sem, contudo, limitar a prática da relação sexual.

As mulheres que não fazem TRH ou sob o uso da isoflavona percebem essa fase sem grandes transformações, sentem-na sem ganhos ou perdas. Referem terem ganhado em relação a 
não terem mais as cólicas, mal estar, não engravidar e mesmo por não terem os sintomas da menopausa, mas realçam as perdas em relação à ausência de oportunidade de ser mãe, destacando o envelhecimento e o aparecimento de dores.

Enquanto as mulheres com TRH referem que o sono melhorou, ressaltam ser essa a melhor fase de sua vida por terem conseguido mais maturidade, terem ganhado mais orientação de saúde com a oportunidade de perguntar nas consultas. Mesmo mulheres que passam por problemas, como de doença crônica do marido, morte de filho etc., declaram sentirem-se bem em suas narrativas.

Unidade temática II: conhecimento sobre propostas terapêuticas na perimenopausa e pós-menopausa

As mulheres com ou sem o uso da TRH relatam ter conhecimento de outras formas de promoção da saúde da mulher na perimenopausa realçando a importância da atividade física por meio da natação, hidroginástica, caminhada, de uma alimentação balanceada, da genética herdada e da medicalização, quando necessária, para manterem o bem estar físico, psíquico e social.

As entrevistadas que não usam a TRH ou ex-usuárias, relacionam o conhecimento dos hormônios com os efeitos adversos.

"Então, estou sem tomar um ano. Agora eu vou passar no doutor ..., para ver o que vão fazer, se vão dar alta. (...) Não explicou, mas a gente entendia, porque pediu mais mamografia, mais vezes, a gente não tinha costume, só tirava de ano em ano" $(4,59, \mathrm{~S} / \mathrm{H})$.

Até 1960 , a TRH era recomendada apenas para tratar sintomas severos do período da perimenopausa; foi em 1966 que se popularizou por causa do lançamento do livro Feminine Forever, pelo médico ginecologista americano Robert Wilson, que fez carreira como garoto-propaganda do hormônio estrogênio. Este livro defendia o estrogênio como tratamento para todos os sintomas das mulheres mais velhas; omitia, entretanto, as ligações do autor com a indústria farmacêutica 23 .

Marinho \& Roquete 24 relatam que é comum ouvir das mulheres, ao investigar no primeiro retorno após a introdução da TRH, respostas do tipo "a gente se sente bem”, “outra pessoa, muito melhor". Mas é preciso não se perder de vista o efeito dominó proporcionado pela TRH, a saber, ao tratar do sintoma físico (vasomotor e atrofia genital) resolva os psíquicos, que muitas vezes são secundários aos físicos.
“Deu, deu, porque os calores acabaram assim na primeira cartela. Tanto que acho que a minha reposição não seria tão necessária agora, porque foi muito rápido, pra mim acabou, até eu quero voltar para ver se eu preciso continuar" (1, 54, C/H).

A suspensão do tratamento, implica perda de massa óssea, assim como nos benefícios da área urogenital. Por outro lado, o uso prolongado se associa a um pequeno aumento da incidência de câncer de mama, e, quanto à proteção cardiovascular, ainda não há dados conclusivos 12,13.

A hormonioterapia na perimenopausa constitui uma realidade não somente para mulheres hígidas, mas também para aquelas portadoras de doenças crônicas que também vivem a sua menopausa 25. É preciso assisti-las com maior atenção porque muitas vezes os sinais e sintomas desse período podem ser confundidos com os da patologia de base: "aí, eu comecei a tomar aquele hormônio sintético, só que como eu já tive problema de mioma, aí ... eu comecei a ter sangramento direto, tomei dois, três meses e foi bem, (...) aí teve que suspender e agora eu tomo hormônio fito... terapêutico, não é? [Isoflavona] Isso ..." $(11,52, \mathrm{~S} / \mathrm{H} / \mathrm{I})$.

A indicação das isoflavonas é feita em decorrência da sua atividade estrogênica fraca, é muito menos potente do que o estrogênio sintético 26 . O consenso de opinião da sociedade norte-americana de menopausa é que, segundo estudo baseado em evidências, o uso da isoflavona na menopausa são inconclusivos 27 .

As narradoras que utilizam ou não a TRH, fazem-no em razão do conhecimento empírico adquirido nas conversas com outras mulheres ou por meio de informação obtida na mídia escrita e falada. Poucas referem algum tipo de conhecimento científico e, quando têm, raramente foi fornecido pelos profissionais da saúde, particularmente pelos médicos. Tal fato, à luz da saúde pública, obriga-nos a refletir sobre a qualidade da assistência oferecida às mulheres na perimenopausa, bem como sobre a medicalização e expropriação da saúde dos corpos femininos nos serviços ofertados.

\section{Considerações finais}

A medicalização de uma condição biológica universal nos fez pensar no motivo de isso vir ocorrendo. O momento histórico na sociedade atual é considerado por alguns estudiosos como pós-moderno. Na pós-modernidade encontramos a desconstrução do curso da vida com a tendência a uma maior flexibilidade e varie- 
dade nestas fases. No campo biológico, não podemos redesenhar o mapa do curso da vida, não podemos eliminar estágios ou colocá-los em outra ordem, porque o curso da vida humana tem como premissa fases precedentes de desenvolvimento.

Nas sociedades emergentes pós-modernas, a fase da mulher na menopausa é apresentada com imagens que a retratam como uma etapa da vida em que a juventude, a vitalidade, a sexualidade e a atratividade podem ser mantidas mediante condutas de promoção de saúde como o estímulo aos exercícios físicos, hábitos alimentares, controle do peso, evitando o tabagismo e outros. Essas mudanças nos hábitos de vida são úteis tanto quanto a reposição hormonal.

Nas narrativas das depoentes a este estudo, houve uma aclamação geral por maiores esclarecimentos e conhecimentos acerca desta fase da vida da mulher nas instituições de saúde e nos outros meios de comunicação. Para encarar esse desafio, vimos, como enfermeira, a necessidade de se ter uma abordagem interdisciplinar na assistência às mulheres na menopausa, por entendermos que se tratar de um con-

\section{Resumo}

Este estudo, uma pesquisa qualitativa, tem por objetivo conhecer o significado da perimenopausa/pós-menopausa e o vivenciar de seus sinais e sintomas para mulheres que usam ou não a terapia de reposição hormonal (TRH). Os dados empíricos foram obtidos por meio de entrevista realizada com 11 mulheres, de 45 a 59 anos de idade, usuárias ou não de TRH e freqüentadoras de diferentes serviços de saúde no Município de São Paulo, Brasil. A análise foi realizada por meio da hermenêutica. Os resultados mais significativos revelam que as mulheres que não fazem uso da TRH apresentam os sintomas da perimenopausa, mas convivem com eles; as mulheres que fazem uso da isoflavona procuram por terapias alternativas para os sintomas que as incomodam; ao passo que as mulheres sob o uso da TRH declaram uma sensação de bem estar. $O$ artigo sugere a importância de capacitar os profissionais da área de saúde para compreenderem que a perimenopausa e a pós-menopausa fazem parte do processo de envelhecimento e, portanto, devem ser consideradas como processo vital e não patológico.

Enfermagem; Envelhecimento; Menopausa; Terapia de Reposição Hormonal; Saúde da Mulher junto de seres humanos em diferentes contextos sociais e culturais.

O princípio de que o uso da TRH deveria ser considerado em toda mulher na perimenopausa e pós-menopausa, o chamado mito wilsoniano, precisa ser desmantelado. É preciso que se faça uma análise cuidadosa individual dos riscos e benefícios, esclarecendo a mulher sobre a terapia a que vai se submeter. Além dos impedimentos de ordem médica, importa avaliar que existem situações que contra-indicam o uso da TRH como o baixo nível de colaboração, dificuldade intelectual, social ou econômica, além da resistência por parte da mulher, mesmo que esclarecidas.

Entendemos ser apropriado o investimento na assistência à saúde e na participação social das mulheres na perimenopausa para que tenhamos na próxima geração de idosos uma população com conhecimento do que é o envelhecer com qualidade de vida. Vale destacar que a geração que hoje está na fase da transição menopáusica fará parte da sexta população do mundo em número de idosos, prevista para 2025 no Brasil.

\section{Colaboradores}

S. M. G. Vigeta foi responsável por toda a pesquisa desde a aquisição dos dados até a interpretação dos mesmos. A. C. P. Brêtas participou de todo o processo do estudo.

\section{Agradecimentos}

Este trabalho recebeu incentivo da Coordenação de Aperfeiçoamento de Pessoal de Nível Superior (CAPES). Integra a linha de pesquisa Fundamentos e Práticas de Enfermagem em Saúde Coletiva do Programa de Pós-Graduação em Enfermagem da Universidade Federal de São Paulo. 


\section{Referências}

1. Netto PM, Ponte JR. Envelhecimento: desafio na transição do século. In: Netto PM, organizador. Gerontologia. São Paulo: Atheneu; 1996. p. 3-12.

2. Marinho R. Visão atual do climatério. In: Marinho $\mathrm{R}$, organizador. Climatério. Rio de Janeiro: Medsi; 2000. p. 1-10.

3. Fundação Instituto Brasileiro de Geografia e Estatística. Censo Demográfico 2001. http://www. ibge.net/brasil (acessado em 01/Jun/2002).

4. Oliveira MC. Menopausa, reposição hormonal e a construção social da idade madura. In: Néri AL, Debert GG, organizadores. Velhice e sociedade. Campinas: Papirus; 1999. p. 69-86.

5. Santos LV. Menopausa - construindo uma nova identidade [Dissertação de Mestrado]. São Paulo: Departamento de Enfermagem, Universidade Federal de São Paulo; 1995.

6. Featherstone M. O curso da vida: corpo, cultura e o imaginário no processo de envelhecimento. In: Debert GG, organizador. Antropologia e velhice. Campinas: Instituto de Filosofia e Ciências Humanas, Universidade Estadual de Campinas; 1994. p. 49-71. (Coleção Textos Didáticos 13).

7. Hardy E, Alves G, Osis MJD. Sociologia do climatério. In: Pinotti JA, Halbe HW, Hegg R, organizadores. Menopausa. São Paulo: Roca; 1995. p. 31-6.

8. Uchôa E. Contribuições da antropologia para uma abordagem das questões relativas à saúde do idoso. Cad Saúde Pública 2003; 19:849-53.

9. Organización Mundial de la Salud. Investigaciones sobre la menopausia en los años noventa. Genebra: Organización Mundial de la Salud; 1996. (Serie de Informes Técnicos 866).

10. Bagnoli VR, Fonseca AM. Etiopatogenia do climatério. In: Sampaio NAP, Fonseca AM, Bagnoli VR, Halbe HW, Pinotti JA, organizadores. Síndromes climatéricas. São Paulo: Atheneu; 1999. p. 9-14.

11. Pinto Neto AM, Pedro AO, Hardy E, Osis MJD, Costa-Paiva LHS, Martinez EZ. Caracterização das usuárias de terapia de reposição hormonal do Município de Campinas, São Paulo. Cad Saúde Pública 2002; 18:121-7.

12. Women's Health Initiative Investigators. Risks and benefits of estrogen plus progestin in healthy postmenopausal women: principal results from the Women's Health Initiative randomized controlled trial. JAMA 2002; 288:321-33.

13. Women's Health Initiative Investigators. Effects of conjugated equine estrogen in postmenopausal women with histerectomy: the Women's Health Initiative randomized controlled trial. JAMA 2004; 291:1701-12.
14. Minayo MCS. Introdução à metodologia de pesquisa social. São Paulo: Editora Hucitec/ABRASCO; 1998.

15. Santos BS. Um discurso sobre as ciências. Porto: Editora Afrontamento; 1998.

16. Thiollent M. Crítica metodológica, investigação social e enquete operária. São Paulo: Polis; 1987.

17. Bordieu P. O poder simbólico. Rio de Janeiro: Bertrand Brasil; 1998.

18. Foucault M. Microfísica do poder. Rio de Janeiro: Graal; 1990

19. Fortes JRA, Hirata ES. Aspectos psiquiátricos do climatério. In: Pinotti JA, Halbe HW, Hegg R, organizadores. Menopausa. São Paulo: Roca; 1995. p. 41-6.

20. Baggio MA. Depressão no climatério. In: Marinho $\mathrm{R}$, organizador. Climatério Rio de Janeiro: Medsi; 2000. p. 113-26.

21. Ferriani RA. Alterações hormonais no climatério repercussões a curto prazo. In: Marinho R, organizador. Climatério. Rio de Janeiro: Medsi; 2000. p. 13-22.

22. Trien SF. Menopausa: a grande transformação. Rio de Janeiro: Rosa dos Tempos; 1994.

23. Greer G. Mulher. Maturidade e mudança. São Paulo: Augustus; 1994

24. Marinho R, Roquete ALB. O ginecologista atendendo a mulher no climatério. In: Marinho R, organizador. Climatério. Rio de Janeiro: Medsi; 2000. p. 155-74.

25. Fonseca AM, Bagnoli VR, Vergolino RVD, Parellada CI, Pinotti JA. Terapia de reposição hormonal: doenças auto-imunes. In: Fonseca AM, Bagnoli VR, Halbe HW, Pinotti JA, organizadores. Terapia de reposição hormonal em situações especiais. Rio de Janeiro: Revinter; 2001. p. 13-22.

26. Rodrigues AA. A importância da nutrição no climatério. In: Marinho R, organizador. Climatério. Rio de Janeiro: Medsi; 2000. p. 343-70.

27. The North American Menopause Society. The role of isoflavones in menopausal health: consensus opinion of the North American Menopause Society. Menopause 2000; 7:215-29.

Recebido em 17/Nov/2003

Versão final reapresentada em 13/Mai/2004

Aprovado em 19/Jul/2004 